\title{
Role of anionic phospholipids in the adaptation of Bacillus subtilis to high salinity
}

Correspondence

Carmen Sánchez-Rivas sanchez@qb.fcen.uba.ar

Received 14 July 2005

Revised 8 November 2005

Accepted 29 November 2005

\author{
Claudia S. López, ${ }^{1,2} \uparrow$ Alejandro F. Alice, ${ }^{1} \dagger$ Horacio Heras, ${ }^{3}$ Emilio A. Rivas ${ }^{2}$ \\ and Carmen Sánchez-Rivas ${ }^{1}$
}

\author{
${ }^{1}$ Facultad de Ciencias Exactas y Naturales de la Universidad de Buenos Aires, Departamento \\ de Química Biológica, Ciudad Universitaria Pabellón II 1428, Buenos Aires, Argentina \\ 2Instituto de Biología Celular y Neurociencias 'Dr E. De Robertis', Facultad de Medicina \\ Universidad de Buenos Aires (UBA), Paraguay 2155 (1121), Buenos Aires, Argentina \\ ${ }^{3}$ Instituto de Investigaciones Bioquímicas de La Plata (INIBIOLP) (UNLP/CONICET), Facultad \\ de Ciencias Médicas, Calle 60 y 120 (1900), La Plata, Argentina
}

\section{INTRODUCTION}

During growth in hyperosmotic media containing elevated concentrations of inorganic or organic solutes, bacteria develop several strategies, including changes in the regulation

In memory of Dr Emilio A. Rivas.

tPresent address: Dept of Molecular Microbiology and Immunology, Oregon Health and Science University, Portland, OR, USA.

Abbreviations: CL, cardiolipin; DGDG, diglucosyldiacylglycerol; GP, generalized polarization; LB, Luria-Bertani medium; LBN, Luria-Bertani medium with $1.5 \mathrm{M} \mathrm{NaCl}$; Laurdan, 2 dimethylamino-6-lauroylnaphthalene; LUVs, large unilamellar vesicles; LysPG, lysylphosphatidylglycerol; $\mathrm{PE}$, phosphatidylethanolamine; $\mathrm{PG}$, phosphatidylglycerol. of metabolic pathways and activation of transporters, to overcome both the salt and the osmotic stresses (Wood, 1999; Poolman et al., 2002). Upon modification of the external osmolality of the media the cells change their volume and, among other properties, their membrane tension. Moreover, it has been suggested that a change in the membrane potential and ion gradients might also occur (Poolman et al., 2004). The osmotic response is characterized by two different phases. Among the first responses to the new environmental conditions, the transport of large amounts of $\mathrm{K}^{+}$from the extracellular medium via turgorresponsive transport systems has been described both in enteric Gram-negative bacteria such as Escherichia coli and 
Salmonella typhimurium and in the Gram-positive Bacillus subtilis (McLaggan et al., 1994; Whatmore \& Reed, 1990). Along with this response, in enteric bacteria glutamate synthesis is activated in order to provide the counterions for the increased positive charges in the cytoplasm (Yan et al., 1996), while in B. subtilis synthesis of proline preferentially occurs (Whatmore et al., 1990). As a second response, both Gram-negative and Gram-positive bacteria accumulate in their cytoplasm large amounts of zwitterionic organic molecules such as glycine betaine, carnitine, choline, proline or ectoine, which are highly soluble molecules, commonly named osmoprotectants (Kempf \& Bremer, 1998; Wood, 1999). These compounds are accumulated either through de novo synthesis or by active transport from the environment, depending on the medium composition (Whatmore et al., 1990). Osmoprotectants like glycine betaine and proline increase the cytoplasmic volume and the free water content of the cells at high salinity; therefore their accumulation allows cell proliferation under adverse environmental conditions (Record et al., 1998). In the case of $B$. subtilis, a family of transporters (OpuA-E) is involved in the transport of these compounds (Kappes et al., 1996; von Blohn et al., 1997).

Until recently studies concerning the adaptation of cells against osmotic stress have been essentially oriented to genetic and physiological regulation aspects (Csonka \& Hanson, 1991; von Blohn et al., 1997; Glassker et al., 1998; Kappes et al., 1996; Price et al., 2001). Recently, a transcriptome analysis of the adaptation of $B$. subtilis cells to high salinity showed that at least 125 genes are induced, thus revealing the complexity of this response (Steil et al., 2003).

The behaviour of bacterial envelopes in the presence of elevated $\mathrm{NaCl}$ concentrations in the environment has not been sufficiently explored, in spite of the important traffic of compounds that takes place between the cytoplasm and the external medium. It is to be expected that the stability and permeability of the cell membranes will play a fundamental role in the adaptation to different kinds of stresses and that these properties would be closely related to lipid and fatty acid composition. The cell membrane is the first barrier to an increased ionic strength, and the sensing mechanism would take place in it (Poolman et al., 2002, 2004). In addition, several sigma factors required for extracytoplasmic functions (membrane and wall synthesis) have been shown to respond to stress effectors (Cao et al., 2002). In particular, Horsburgh \& Moir (1999) showed that in B. subtilis, the sigM gene product is required for normal cell growth under salt stress. Moreover, the SigM protein is involved in maintaining the membrane and cell wall integrity in response to different stresses in exponential growth phase and is activated by these stresses (Thackray \& Moir, 2003).

Although B. subtilis cells are able to resist environmental osmotic changes, in our laboratory we have demonstrated that the osmotic response in this bacterium is mainly a stationary-phase-regulated event that shares common regulators with the sporulation process, DegU-P being the positive regulator (Ruzal \& Sánchez-Rivas, 1994, 1998). We have also found that during growth in hypersaline medium $B$. subtilis cells develop an asymmetric septum similar to that developed by cells in sporulation stage II (Ruzal et al., 1998). However, in these cells the sporulation process is inhibited (Ruzal \& Sánchez-Rivas, 1994).

We have been studying for several years the biochemical and biophysical characteristics of the cell envelopes of cells grown under osmotic stress both in B. subtilis and in Lactobacillus casei (López et al., 1998, 2001, 2002; Piuri et al., 2003; Machado et al., 2004; Piuri et al., 2005). In these studies, the analysis of membrane lipids has revealed that there is a significant increase in the anionic lipid fraction such as phosphatidylglycerol (PG) and especially cardiolipin (CL) when B. subtilis cells are grown in LB medium containing $1.5 \mathrm{M} \mathrm{NaCl}$. We have also described an important modification in the fatty acid composition due to this stress (the proportion of straight-chain saturated fatty acids increases while that branched-chain saturated fatty acids decreases). These variations are accompanied by a higher membrane microviscosity (López et al., 2001, 2002). Similar variations in the CL content have also been described in protoplasts of B. subtilis due to a polyethylene glycol stress (Sánchez-Rivas \& Bohin, 1983), in Rhodobacter sphaeroides (Catucci et al., 2004) and in Mesorhizobium loti grown under acidic stress (Correa et al., 1999), thus suggesting that an increased CL content might be a general cellular response due to membrane adjustments.

The in silico examination of the B. subtilis genome sequence has revealed the presence of three putative CL synthases ( $y w n E$, $y w i E$ and $y w j E$ ). In addition, a transcriptional microarray analysis of stress responses has indicated that the $y w i E$ gene is induced during ethanol or $\mathrm{NaCl}$ stress, its promoter being sigma B-dependent, as for most of the genes responding to general stress effectors (Price et al., 2001; Petersohn et al., 2001). Moreover, recently Kawai et al. (2004) showed that in the B. subtilis Marburg strain, $y w n E$ is the gene that plays the main role in CL synthesis and therefore renamed it as clsA.

Since our previous experiments showed that there are important changes in the B. subtilis membrane composition due to elevated $\mathrm{NaCl}$ concentrations in the medium, and in particular in the CL content, we decided to investigate the role of the three putative CL synthase genes in our strain context (YB886 derivative of strain 168 cured of prophages: Yasbin et al., 1980). In addition, a conditional mutant ( $p g s A$ ) for synthesis of PG, the precursor of CL, was also studied. Physiological studies of hyperosmotic adaptation and membrane biochemical behaviour of these mutant strains are reported here.

\section{METHODS}

Strains, plasmids and primers. These are listed in Table 1.

Bacterial growth, osmoresistance and sporulation. B. subtilis strains were grown with vigorous agitation at $37^{\circ} \mathrm{C}$, in Luria-Bertani 
Table 1. Bacterial strains, plasmids and primers

\begin{tabular}{|c|c|c|}
\hline & Characteristics & Source/reference \\
\hline \multicolumn{3}{|c|}{ Bacterial strains } \\
\hline \multicolumn{3}{|c|}{ B. subtilis } \\
\hline YB886 & $\operatorname{trpC} 2$ metB5 SP $\beta(S)$ xin-1 & Yasbin et al. (1980) \\
\hline CSL1 & YB886, ywiE::pMutin-4 & This study \\
\hline CSL2 & YB886, ywnE::pMutin- $4^{*}$ & This study \\
\hline CSL3 & YB886, ywjE::pMutin-4 & This study \\
\hline CSL21 & YB886, amy::pCL31 & This study \\
\hline PGSA1 & YB886, pgsA::pMutin-4 & This study \\
\hline SDB110 & 168, pgsA::pMutin-4 & K. Matsumoto $\ddagger$ \\
\hline \multicolumn{3}{|l|}{ E. coli } \\
\hline TG1 & $\begin{array}{l}\text { supE hsd } \Delta 5 \text { thi } \Delta(\text { lac-proAB }) \\
\mathrm{F}^{\prime}\left(\operatorname{traD} 36 \text { proAB }^{+} \text {lacl }^{\mathrm{q}} \text { lacZ } \Delta \mathrm{M} 15\right)\end{array}$ & Laboratory stock \\
\hline \multicolumn{3}{|l|}{ Plasmids } \\
\hline p-Mutin 4 & $\mathrm{Amp}^{\mathrm{R}} \mathrm{Erm}^{\mathrm{R}}$ & Vagner et al. (1998) \\
\hline p-GEM-T & Cloning vector & Promega \\
\hline pEC20 & pMutin-4:: ywnE $E^{\star}$ & P. Stragier $\neq$ \\
\hline pEC6 & Integrative at amy $\left(\mathrm{Amp}^{\mathrm{R}} \mathrm{Erm}^{\mathrm{R}}\right)$ & Martin-Verstraete et al. (1992) \\
\hline pCL31 & pEC6:: (PywnE-lacZ)* & This study \\
\hline pCL29 & pMutin-4:: ywiE & This study \\
\hline pywjE & pMutin-4:: ywjE & F. Hullo $\ddagger$ \\
\hline Primers & Sequence $\dagger$ & \\
\hline $\mathrm{C} 1$ & 5' -ATAAAGCTTAAAGGGAAAGCTGTTTAAG-3' & This study \\
\hline $\mathrm{C} 2$ & 5'-ATAGGATCCTCTCTGCTGATATATTC-3' & This study \\
\hline C5 & 5'-GGTTTTAAAGAATTCTGCCCG-3' & This study \\
\hline C6 & 5'-TTTGGATCCTAATTCATCGCC-3' & This study \\
\hline C7 & 5'-ATTCTGCTTGATATCTTCATG-3' & This study \\
\hline PGS & 5'-AAATCACACTAGCTAGAATCGC-3' & This study \\
\hline $\mathrm{LZ}$ & 5'-AGTACAGCGCGGCTGAAATC-3' & This study \\
\hline
\end{tabular}

${ }^{\star}$ The $y w n E$ sequence corresponds to the $c l s A$ gene according to Kawai et al. (2004) and the present work. $\dagger$ The underlined sequences indicate restriction sites.

$\ddagger$ K. Matsumoto, Dept of Biochemistry and Molecular Biology, Saitama University, Sakura, Saitama, Japan; P. Stragier, IBPC-CNRS, Rue P. et M. Curie, Paris V, France; F. Hullo, Institut Pasteur, Unité de Génétique des genomes bactériens, 28 Rue du Dr Roux, Paris XV, France.

(LB, tryptone $20 \mathrm{~g} \mathrm{l}^{-1}$, yeast extract $10 \mathrm{~g} \mathrm{l}^{-1}, \mathrm{NaCl} 10 \mathrm{~g} \mathrm{l}^{-1}$ ) medium (control) or LB medium supplemented with $1.5 \mathrm{M} \mathrm{NaCl}$ final concentration (LBN) unless otherwise stated in the text. Solid media were obtained by adding $1.5 \%(\mathrm{w} / \mathrm{v})$ agar. The antibiotics used were erythromycin (Erm; $1 \mu \mathrm{g} \mathrm{ml}^{-1}$ ) and chloramphenicol $\left(5 \mu \mathrm{g} \mathrm{ml}^{-1}\right)$.

Growth curves were obtained from cultures grown overnight in aerated LB medium at $37^{\circ} \mathrm{C}$. These cultures were diluted (1/50) in fresh LB medium or LB medium containing different concentrations of $\mathrm{NaCl}$ as indicated in the figures. Cellular growth was monitored by measuring $\mathrm{OD}_{600}$.

For the osmoresistance assays on solid media, exponential or early stationary phase cultures grown in LB medium were diluted and plated onto LB (control) and LBN plates and incubated for $2-3$ days at $37^{\circ} \mathrm{C}$. Osmoresistance is expressed as the percentage of c.f.u. on LBN relative to the control.

The sporulation efficiency was determined from cultures grown in Schaeffer's sporulation medium (Schaeffer et al., 1965) incubated at $37^{\circ} \mathrm{C}$ for $48 \mathrm{~h}$. Serial dilutions in $0.85 \% \mathrm{NaCl}$ were heat-treated at
$70{ }^{\circ} \mathrm{C}$ for $10 \mathrm{~min}$ and plated on LB for c.f.u. determination. As control, non-heated cells were also diluted and plated and the percentage sporulation efficiency was calculated as (c.f.u. heat-treated/c.f.u. control) $\times 100$.

Construction of ywiE, ywnE, ywjE and pgsA mutants in $B$. subtilis YB886. The $y w i E$, $y w n E$ and $y w j E$ mutant strains were constructed by using the suicide plasmid pMutin-4 (Vagner et al., 1998) harbouring the promoterless lac $Z$ gene.

Chromosomal DNA and plasmid isolations were performed according to Bron (1990). The strain mutated in the $y w i E$ gene was constructed as follows. DNA from strain YB886 was used as template for PCR amplification using primers $\mathrm{C} 1$ and $\mathrm{C} 2$ (Table 1) and containing additional EcoRI or BamHI sites. This fragment was ligated to pMutin-4 previously digested with the same enzymes. The resulting plasmid, pCL29, was used to transform B. subtilis YB886 competent cells prepared as indicated by Kunst \& Rapoport (1995). Since the amplicon contains an internal segment of the $y w i E$ gene, $\mathrm{Erm}^{\mathrm{R}}$ colonies raised by homologous recombination led to a knockout mutation. The recombinants were checked by Southern blotting (data not shown) and one containing the expected construction, named CSL1, was further studied. 
The strain with a mutation in the $y w n E$ gene was obtained by using plasmid pEC20 (Table 1), which contains an internal fragment of the $y w n E$ gene cloned into pMutin-4. The mutant strain in the $y w j E$ gene was obtained using the plasmid pywjE (Table 1). These plasmids were used to transform B. subtilis YB886 competent cells and the transformants were selected on plates containing Erm as described above. The Erm ${ }^{\mathrm{R}}$ colonies were analysed by PCR using primers C5 and LZ (Table 1) for the $y w n E$ mutant strain and primers C7 and LZ (Table 1) for the $y w j E$ mutant strain. Colonies identified with the corresponding mutation were named CSL2 $(y w n E)$ and CSL3 $(y w j E)$.

Strain CSL21 was constructed as follows. A fragment containing part of the putative $y w n E$ promoter (nucleotides -143 to +460 relative to the ATG) was amplified by standard PCR using primers C5 and C6 (Table 1) and cloned into pGEM-T. The resulting plasmid was subsequently digested using EcoRI and BamHI and the insert was purified and subcloned into the pEC6 suicide vector previously digested with the same enzymes. The resulting plasmid was named pCL31 (Table 1). Using this strategy the $y w n E$ promoter was fused to the lac $Z$ gene, generating a transcriptional fusion flanked by regions with homology to the amy locus in B. subtilis. pCL31 was then introduced by transformation into B. subtilis competent cells. Since this plasmid cannot replicate in this species, recombinants in which homologous recombination within the amy locus had occurred were selected on plates containing chloramphenicol and screened by their failure to degrade starch. One of them was chosen, named CSL21, and analysed.

The $p g s A$ mutation was transferred from strain SDB110 into the wildtype strain YB886 by transformation using chromosomal DNA from strain SDB110. In this construction the $p g s A$ gene is under the control of the Pspac promoter, while the lac $Z$ gene is under the control of the $p g s A$ promoter. Selection of recombinants was performed on plates supplemented with erythromycin and $0.5 \mathrm{M}$ IPTG. Several colonies were analysed by PCR using primers PGS and LZ (Table 1), allowing the selection of the mutant PGSA1.

$\boldsymbol{\beta}$-Galactosidase determination. $\beta$-Galactosidase activity was determined as described by Miller (1992) but samples were permeabilized by treatment with lysozyme $\left(10 \mathrm{mg} \mathrm{ml}^{-1}\right)$ and sodium deoxycholate $(0.01 \%, \mathrm{w} / \mathrm{v})$ at $37^{\circ} \mathrm{C}$ for $30 \mathrm{~min}$ before the assay.

Lipid extraction and purification. Cultures of the different strains grown under specific conditions as described in each figure were harvested by centrifugation and the pellets used for lipid extraction. Total lipids were extracted according to the original method of Rivas \& Luzzati (1969) with some modifications as described by López et al. (1998). Polar lipids were separated from neutral lipids by preparative TLC run in hexane/ethyl ether/acetic acid $(80: 20: 1$, by vol.). The fractions corresponding to the polar lipids were scraped off and extracted with chloroform/methanol $(2: 1, \mathrm{v} / \mathrm{v})$. The resulting suspension was shaken and filtered according to Kates (1986). This procedure was repeated twice. Polar lipids were analysed by HPTLC (Merck Art. 5074), in chloroform/methanol/acetic acid $(65: 25: 6$, by vol.). Phospholipids and glycolipids were detected with iodine vapour and identified by comparison with authentic purified standards and by treatment with specific stains. Zones from the plates corresponding to each phospholipid fraction were scraped off according to Kates (1986) and quantified. Lipid phosphorus was determined according to Bartlett (1959), and total carbohydrates were quantified as indicated by Hanson \& Phillips (1981).

Fatty acid analysis. Total lipid extract was suspended in $2 \mathrm{ml}$ of a fresh solution of $\mathrm{KOH} 10 \%(\mathrm{w} / \mathrm{v})$ in ethanol and digested at $80^{\circ} \mathrm{C}$ for $45 \mathrm{~min}$. Distilled water $(1 \mathrm{ml})$ was added and the aqueous solution extracted three times by shaking with $2 \mathrm{ml}$ hexane. The upper organic phase was discarded and the aqueous layer was acidified with $0.5 \mathrm{ml}$ concentrated $\mathrm{HCl}$ and extracted twice with $2 \mathrm{ml}$ hexane.
Free fatty acids were dried under a nitrogen stream and submitted to methyl esterification with $2 \mathrm{ml} 2 \% \mathrm{H}_{2} \mathrm{SO}_{4}$ in methanol at $60^{\circ} \mathrm{C}$ for $120 \mathrm{~min}$ under a nitrogen atmosphere. Then $2 \mathrm{ml}$ chloroform and $0.67 \mathrm{ml}$ distilled water were added. The aqueous phase was discarded and the organic phase was washed twice with distilled water. After washing, the organic phase was evaporated under a nitrogen stream, resuspended in hexane, and fatty acid methyl esters analysed by gas chromatography using a GLC-FID HP6890 capillary GC (Hewlett Packard) with an Omegawax Capillary column (30 $\mathrm{m} \times 250 \mu \mathrm{m}, 25 \mu \mathrm{m}$ nominal) (Supelco 11090-02A). As a standard a mixture of bacterial fatty acid methyl esters was used (Bacterial Acid Methyl Esters Mix 4780-U, Supelco).

Isolation of large unilamellar vesicles (LUVs) and membranes. Multilamellar lipid vesicles were prepared by mixing in glass tubes appropriate amounts of stock solutions of total lipids at $1 \mathrm{mM}$ in chloroform/methanol $(2: 1, \mathrm{v} / \mathrm{v})$ and the fluorescent probe. The solvent was evaporated by nitrogen flux and the dried samples were suspended in buffer (sucrose $0 \cdot 25 \mathrm{M}$, EDTA $1 \mathrm{mM}, \mathrm{pH} 7 \cdot 0$ ), and vortexed vigorously for $5 \mathrm{~min}$. These multilamellar vesicles were extruded through a polycarbonate filter $(100 \mathrm{~nm}$ pore diameter mounted in a miniextruder (Avestin) fitted with two $0.5 \mathrm{ml}$ Hamilton syringes. To obtain the LUVs, the samples were subjected to 13 passes through two filters in tandem. The membranes were isolated according to Lysenko et al. (1997). A membrane suspension $(1 \mathrm{mM})$ in $0.25 \mathrm{M}$ sucrose and $1 \mathrm{mM}$ EDTA pH $7 \cdot 0$ was mixed with the fluorescent probe and incubated at room temperature for $30 \mathrm{~min}$.

Fluorescence spectroscopy. 2-Dimethylamino-6-lauroylnaphthalene (Laurdan) was purchased from Molecular Probes. The stock solution was prepared in methanol and the final Laurdan probe/ lipid phosphorus ratio was $1 / 400$. To calculate the generalized polarization (GP), different Laurdan emission spectra were acquired by using a fixed excitation wavelength of 330 and $410 \mathrm{~nm}$. The Laurdan excitation spectra were also obtained at two different wavelengths (435 and $490 \mathrm{~nm}$ ) and the four different series of resulting spectra were used to calculate the GP according to Parasassi et al. (1994). For each sample ten excitation and emission spectra were acquired and corrected for the background by subtracting the signal of unlabelled samples. The parameter calculated was $\mathrm{GP}_{\mathrm{exc}}=$ $\left(I_{435}-I_{490}\right) /\left(I_{435}+I_{490}\right)$, where $I_{435}$ and $I_{490}$ are the intensities at each excitation wavelength of 330 and $410 \mathrm{~nm}$, using the fixed emission wavelengths of 435 and $490 \mathrm{~nm}$, respectively. The hydration level was calculated as the $\mathrm{GP}_{\text {exc }}$ value obtained at $350 \mathrm{~nm}$ (Hirsch-Lerner \& Barenholz, 1999).

\section{RESULTS}

\section{Polar lipids of the wild-type, ywiE, ywnE and ywjE mutant strains}

The polar lipid composition of the wild-type YB886, CSL1 (ywiE), CSL2 (ywnE) and CSL3 (ywjE) strains grown in LB medium was analysed by HPTLC. The lipid profile and percentage of the main polar lipids obtained for the CSL1 and CSL3 strains was similar to that obtained with the wildtype strain, indicating that there was no change in the content of the major polar lipids in these mutant strains (data not shown). However, during the exponential growth phase the strain harbouring the interrupted $y w n E$ gene (CSL2) showed a complete absence of CL and a major increase in PG content compared to its wild-type control (50\% and $25 \%$ of total phospholipids respectively) (Fig. 1a). Concerning the other two phospholipids present 


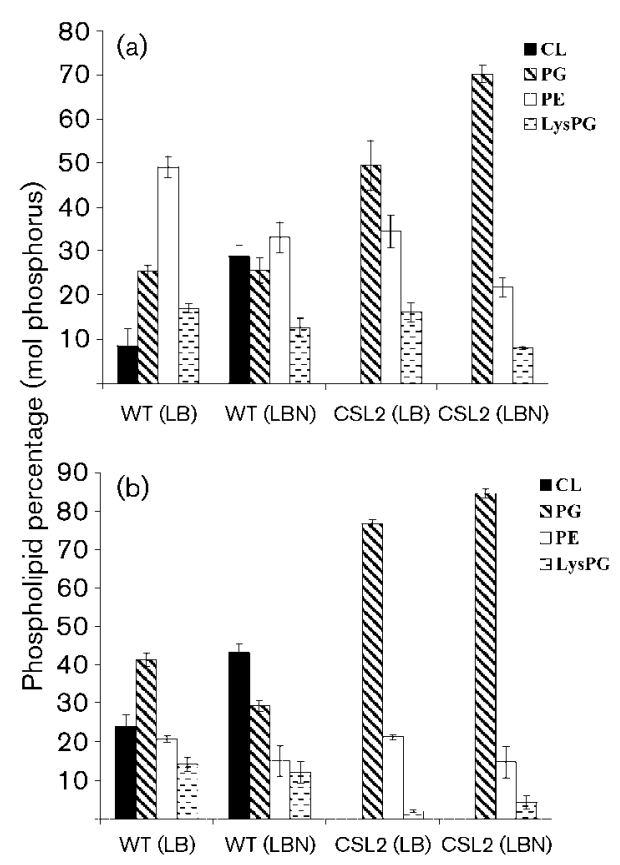

Fig. 1. Phospholipid composition of B. subtilis wild-type YB886 and CSL2 mutant strains in (a) exponential phase and (b) stationary phase. The strains were grown in LB and LBN and phospholipids were determined as indicated in Methods. The values represent the mean \pm SD of three independent experiments.

in this B. subtilis strain, the content of phosphatidylethanolamine (PE) of the CSL2 mutant strain showed a $1 \cdot 4$-fold decrease compared to the wild-type; however, no changes were observed for lysylphosphatidylglycerol (LysPG).

In order to verify if the changes in phospholipid composition described above were specific to the growth phase, we performed the same type of analysis for the wild-type and CSL2 mutant strains grown to stationary phase (Fig. 1b). Interestingly, the results obtained for the wild-type strain indicated that there was an increase of CL in its membrane when the cells reached stationary phase, rising from $8 \%$ to $24 \%$, suggesting that the presence of CL in the B. subtilis membrane is not only dependent on the composition of the media, as we previously described (López et al., 1998), but also on the growth phase (Fig. 1b). In the case of the CSL2 mutant strain, we confirmed that independently of the growth phase (exponential or stationary), this mutant is unable to synthesize CL. Interestingly, we also observed an increased PG content in the CSL2 mutant cells when they reached the stationary phase, rising from $50 \%$ to $80 \%$, while the content of the other two phospholipids, PE and LysPG, decreased (Fig. 1a, b).

These results clearly show that the interruption of the $y w n E$ gene resulted in a complete failure to synthesize the anionic phospholipid CL in the B. subtilis YB886 strain context at both stationary and exponential phase. Since this gene encodes the principal CL synthase in this bacterium, from now on, in accordance with Kawai et al. (2004), we will call it $\operatorname{cls} A$.

\section{Growth at high $\mathrm{NaCl}$ concentration and sporulation efficiency}

Since we have previously demonstrated that there is a major increase in the CL content in the B. subtilis cells during osmotic stress (López et al., 1998), we wondered whether the absence of this phospholipid would have any effect on the osmotic adaptation. Thus, we obtained growth curves for the CSL2 mutant strain in LB medium supplemented with different concentrations of $\mathrm{NaCl}$ ranging from 0.375 to $1.5 \mathrm{M}$. As shown in Fig. 2, at $0.75 \mathrm{M}$ and $1.0 \mathrm{M} \mathrm{NaCl}$, no differences were observed between the wild-type and CSL2 strains in the lag period, growth rate or maximal $\mathrm{OD}_{600}$ reached. In the presence of $1.25 \mathrm{M} \mathrm{NaCl}$, the lag period of the CSL2 mutant strain increased to $8 \mathrm{~h}$, and with $1.5 \mathrm{M}$ $\mathrm{NaCl}$ it increased to $12 \mathrm{~h}$. After this extended lag period in the presence of $1.5 \mathrm{M} \mathrm{NaCl}$, the CSL2 strain started to grow, reaching final $\mathrm{OD}_{600}$ values $50 \%$ lower than the wild-type strain (data not shown). This result clearly indicates that the absence of CL in the B. subtilis membranes, although important for the adaptation to high salinity, is not essential for growth under this condition.

To further investigate the osmoresistance response of the CSL2 strain, cultures were submitted to the plating assay already used in previous studies (Ruzal \& Sánchez-Rivas, 1994). Since these studies have shown that the physiological state of the cells is important to withstand the osmotic stress, we analysed the osmotic response of exponential and early stationary phase cultures grown in LB medium. Although we observed that on $\mathrm{LB}$ plates containing $1.5 \mathrm{M} \mathrm{NaCl}$ the colonies of the CSL2 mutant strain appeared later than those of the wild-type strain, after $48 \mathrm{~h}$ incubation at $37^{\circ} \mathrm{C}$ their size was similar. These results suggest that the delayed growth of the CSL 2 colonies on LBN was due to an extended lag phase, as observed in the growth curves described in Fig. 2. The results obtained in the plate assay (Table 2) indicated that both strains showed the same osmoresistance under the two physiological conditions tested. In addition, as was previously reported, stationary-phase cultures were better able to cope with the ionic strength than exponentialphase cultures, for both the wild-type and the CSL2 mutant.

The sporulation efficiency of the wild-type and CSL2 mutant strains was assayed in Schaeffer's sporulation medium as indicated in Methods. The values obtained for the CSL2 mutant strain were similar to those of the wildtype ( $85 \%$ and $75 \%$, respectively), indicating that the $c l s A$ mutation did not affect the sporulation process.

\section{Phospholipid characterization of the CSL2 cardiolipin null mutant grown in $1.5 \mathrm{M} \mathrm{NaCl}$}

Our results showed that the CSL2 strain, which cannot synthesize CL, was able to grow even at elevated $\mathrm{NaCl}$ concentrations $(1.5 \mathrm{M} \mathrm{NaCl})$ after an extended lag period of 


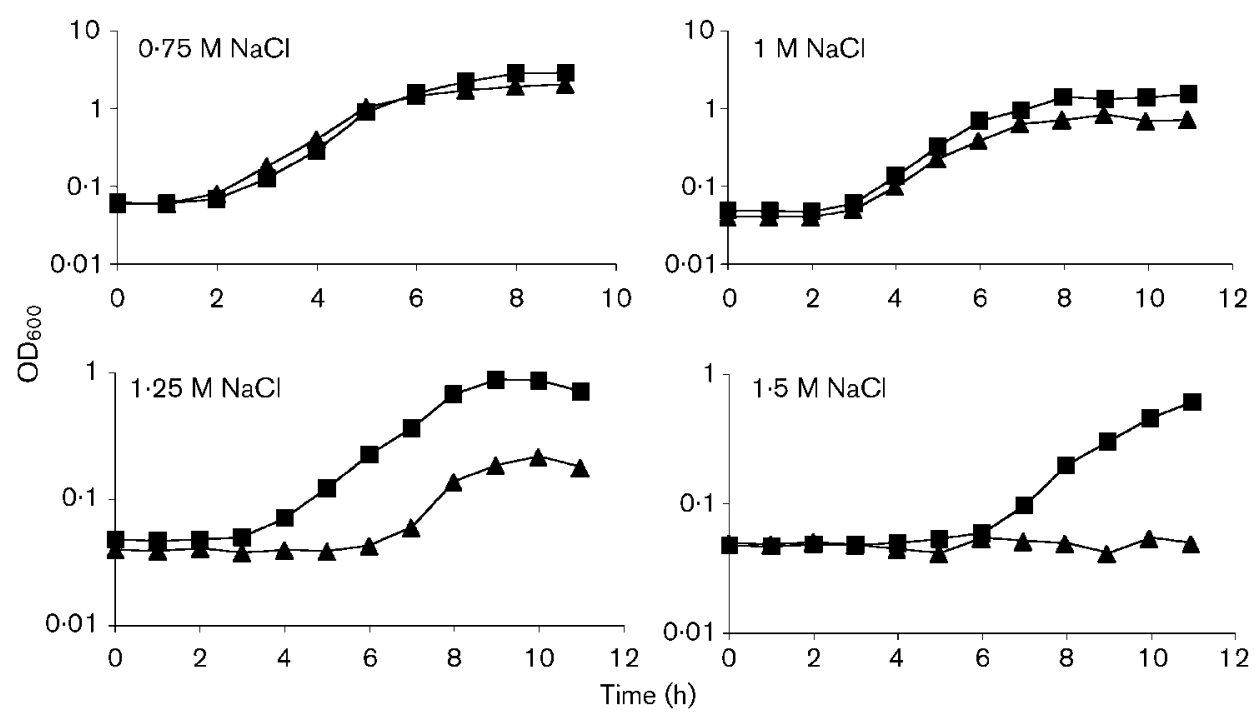

Fig. 2. Growth curves of $B$. subtilis wild-type YB886 ( $\boldsymbol{\square})$ and mutant CSL2 ( $\mathbf{A})$ in LB medium supplemented with different $\mathrm{NaCl}$ concentrations. Overnight cultures in LB broth were diluted $(1: 50)$ into fresh LB broth supplemented with $0 \cdot 75,1 \cdot 0$, 1.25 and $1.5 \mathrm{M} \mathrm{NaCl}$, and incubated in aerated conditions at $37^{\circ} \mathrm{C}$.

12 h (Fig. 2). However, recently Kawai et al. (2004) reported that in B. subtilis Marburg a triple mutant of the cls homologous genes contains a significant amount of CL during the sporulation phase. In the same report, these authors claimed that in their strain background, this phospholipid could be synthesized by the product of the phosphatidylserine synthase gene (pss). Consequently, at least two plausible hypotheses would account for our results: (i) the anionic phospholipid CL is only important for the adaptation of the cells to the new environmental condition but not for their growth (as observed in Fig. 2), or (ii) during the growth of strain CSL2 under high ionic strength conditions the other homologous genes became functional and $\mathrm{CL}$ is synthesized. In order to challenge these hypotheses, the phospholipid composition of strain CSL2 grown in LBN was analysed and

Table 2. Osmoresistance determinations on LB plates supplemented with different $\mathrm{NaCl}$ concentrations

Strains were cultured in aerated LB medium, and at exponential or early stationary phase, samples were diluted and plated on LB or on $\mathrm{LB}$ with $1 \mathrm{M}$ or $1.5 \mathrm{M} \mathrm{NaCl}$. After 2 days incubation at $37^{\circ} \mathrm{C}$, c.f.u. were counted and osmoresistance calculated as indicated in Methods. WT, wild-type YB886.

\begin{tabular}{|lcc|}
\hline \multirow{2}{*}{ Strain (growth phase) } & \multicolumn{2}{c|}{ Percentage osmoresistance in: } \\
\cline { 2 - 3 } & LB + 1 $\mathbf{~ M ~ N a C l}$ & LB+1·5 $\mathbf{~ M ~ N a C l}$ \\
\hline WT (exponential) & $5 \cdot 0$ & $1 \cdot 5$ \\
WT (early stationary) & 94 & 80 \\
CSL2 (exponential) & $8 \cdot 0$ & $1 \cdot 0$ \\
CSL2 (early stationary) & 99 & 84 \\
\hline
\end{tabular}

compared with that obtained in LB. As shown in Fig. 1(a, b), strain CSL2 did not show any detectable CL in either of the different media or growth phases (exponential and stationary), leading us to confirm that no other gene or pathway would supply CL in this B. subtilis strain. In addition, we observed that the PG content in strain CSL2 grown in $1.5 \mathrm{M} \mathrm{NaCl}$ increased both in exponential and in stationary phase, when compared with the same strain grown in normal LB medium (Fig. 1a, b).

\section{Fatty acid and diglucosyldiacylglycerol composition of the CLS2 cardiolipin null mutant}

To determine whether the mutation in the $c l s A$ gene affected other membrane lipid components able to replace the CL deficiency, both fatty acids and diglucosyldiacylglycerol (DGDG) were quantified. It is worth mentioning that DGDG is the only glycolipid present in membranes of $B$. subtilis YB886 as described previously (López et al., 1998). Our results indicated that the CSL2 mutant strain grown in LBN showed a $1 \cdot 6$-fold increase in the glycolipid content when compared to the same strain grown in LB medium. These results are in agreement with the changes in DGDG content previously described for the wild-type strain grown under the same conditions (data not shown and López et al., 1998).

Regarding the fatty acid composition, the branched-chain fatty acids are the major components that esterify the membrane lipids in both the wild-type and CSL2 strains (Fig. 3; Kaneda, 1977; López et al., 1998). The fatty acid profile of the wild-type and mutant strains grown in LB showed a significant difference in the straight-chain saturated fatty acids and in the branched-chain iso series; while in LBN we observed a significant difference in the 


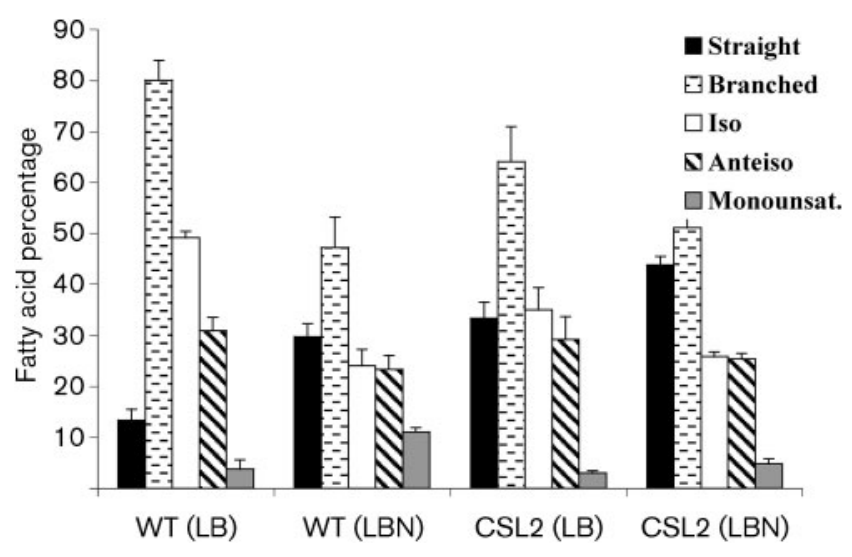

Fig. 3. Fatty acid composition of the $B$. subtilis wild-type YB886 and CSL2 mutant strains grown to stationary phase in LB and LBN. The fatty acids were analysed as indicated in Methods. The values represent the mean \pm SD of three independent experiments. Straight, straight-chain saturated fatty acids; Branched, branchedchain saturated fatty acids; Iso, branched-chain saturated fatty acids iso series; Anteiso, branched-chain saturated fatty acids anteiso series; Monounsat, monounsaturated fatty acids. The fatty acid composition of the wild-type versus the mutant strain in each medium was compared using the Student $t$-test.

monounsaturated fatty acids and, interestingly, also in the straight-chain saturated ones. Altogether, these results suggested that these changes are specifically related to the absence of CL in the membranes of the CSL2 mutant strain.

It is clear from Fig. 3 that the fatty acid composition (specifically straight-chain and branched-chain iso series) of the wild-type strain changes drastically upon increasing the $\mathrm{NaCl}$ concentration. In contrast, little variation in the content of these fatty acids is observed in the CSL2 mutant when the two growth conditions are compared (LB vs LBN). In this context, it is important to note that the straight-chain and branched-chain saturated fatty acid content in the CSL2 strain grown in LB is similar to that of the wild-type strain in LBN (Fig. 3). These results might explain the small variations observed in the content of these fatty acids when the mutant was grown in the two media (compare CSL2 LB vs WT LBN and CSL2 LB vs CSL2 LBN).

These results clearly indicate that in the CL null mutant strain, not only phospholipids but also other membrane components were modified due to the absence of this anionic lipid, suggesting that these modifications could influence physical characteristics of the membrane such as fluidity, generally involved in adaptation to stresses.

\section{Interface properties and lateral lipid packing of the membranes and LUVs of the CSL2 cardiolipin null mutant}

The fluorescent characteristics of the Laurdan probe were used to study the interface properties of LUVs and isolated membranes of the wild-type and CSL2 strains. Laurdan is a fluorescent probe that shows no preferential phase partitioning between ordered and disordered lipid phases and is considered to have uniform lateral and transbilayer distribution (Bagatolli \& Gratton, 2001). Moreover, it has low affinity for proteins in the membrane; therefore this probe is a good reporter molecule to sense molecular relaxation dynamics of solvent dipoles in the membrane as a whole (Parasassi \& Gratton, 1995).

The observed wavelength-dependence pattern of the generalized polarization (GP) of LUVs made from total lipids extracted from both strains (studied at $10{ }^{\circ} \mathrm{C}, 25^{\circ} \mathrm{C}$, $37^{\circ} \mathrm{C}$ and $45^{\circ} \mathrm{C}$ ) changed gradually: the emission $\left(\mathrm{GP}_{\mathrm{em}}\right)$ toward a negative slope and the excitation $\left(\mathrm{GP}_{\text {exc }}\right)$ toward a positive slope, indicating that the lipids were in a liquid ordered state (data not shown and López et al., 2002). In addition, no phase transitions or phase coexistence were detected in both either type of LUVs. The GP $\mathrm{exc}_{\mathrm{e}}$ measured at $350 \mathrm{~nm}$ was taken as indicative of the hydration level of the lipid bilayer at the glycerol moiety of the phospholipids (Hirsch-Lerner \& Barenholz, 1999). The absolute $\mathrm{GP}_{\mathrm{exc}}$ values obtained for the LUVs of the CSL2 strain grown in LBN were higher than those obtained for the rest of the samples. Since higher $\mathrm{GP}_{\text {exc }}$ values indicate a lower degree of dipolar relaxation due to either a lower hydration or water mobility at the polar/non-polar interface, our results suggest an increased lipid packing in this sample (Fig. 4a). At

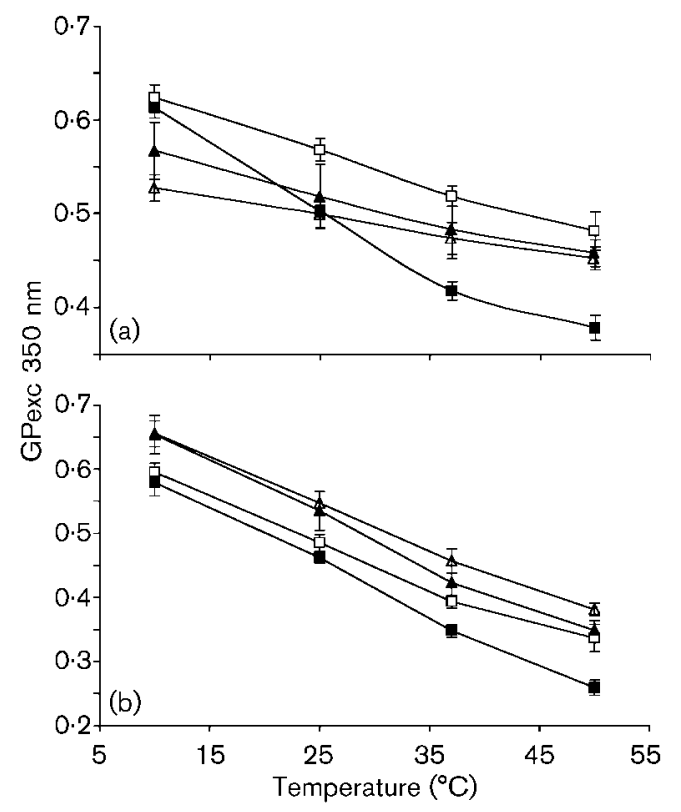

Fig. 4. Generalized polarization $\left(\mathrm{GP}_{\mathrm{exc}}\right)$ of LUVs and membranes. $\mathrm{GP}_{\text {exc }}$ values were calculated at $350 \mathrm{~nm}$ and at the different temperatures with samples from LUVs (a) and isolated membranes (b) obtained from the wild-type (triangles) and CSL2 (squares) cultures in LB (filled symbols) and LBN (open symbols). The values represent the mean \pm SD of four independent experiments. 
physiological temperature $\left(37^{\circ} \mathrm{C}\right)$, the hydration rate will follow the order CSL2 $>\mathrm{WT} \sim \mathrm{WT}+\mathrm{NaCl}>\mathrm{CSL} 2+\mathrm{NaCl}$, LUVs of CSL2 in LB being those presenting the highest interface hydration rate and the lowest lateral lipid packing. Therefore, the changes described in this paper regarding the composition of the membrane (polar lipids and fatty acids) in the CL null mutant undoubtedly lead to changes in the biophysical properties of the LUVs. Moreover, the $\Delta \mathrm{GP}_{\mathrm{exc}}$ of the samples at the different temperatures (data not shown) indicated that the CSL2 LUVs showed more variation of the $\mathrm{GP}_{\text {exc }}$ values within the temperature range studied, these results being related not only to the lipid composition shown in Fig. 1(b) but also to the fatty acid content of the sample shown in Fig. 3.

In order to determine the influence of the membrane proteins on the biophysical properties of the membranes, the same parameters were studied in isolated membranes of both strains. The wavelength dependence pattern of the samples observed within the $25-45{ }^{\circ} \mathrm{C}$ temperature range was characteristic of a liquid ordered state, showing a blue band at $\sim 430 \mathrm{~nm}$ as was observed for the LUVs (data not shown). As in the case of the LUVs, no phase transitions or coexistence were detected in the membrane preparations. However, the red excitation band observed at $380 \mathrm{~nm}$ for the isolated membranes was higher when compared with that of the LUVs (data not shown). This could be due to changes in the lipid packing and/or the polarity of the environment in which the probe is embedded. At the temperatures studied, the absolute $\mathrm{GP}_{\mathrm{exc}}$ values obtained for the isolated membranes of strain CSL2 grown in LBN were higher when compared with the same strain grown in LB, suggesting that in the former membranes there is a lower dipolar relaxation of the probe and/or higher lateral lipid packing (Fig. 4b).

The $\Delta \mathrm{GP}_{\text {exc }}$ calculated as a function of the temperature indicated that the membranes of CSL2 grown in LB showed the highest dependence of this parameter within the temperature range studied (data not shown). However, similar values were obtained for the wild-type and strain CSL2 grown in LBN. These results verified that membrane proteins keep the membrane more sensitive to temperature changes than protein-free-lipid vesicles do. The sharp slopes shown in Fig. 4(b) indicate that in natural membranes, Laurdan revealed a greater $\mathrm{GP}_{\mathrm{exc}}$ dependence on temperature than that obtained in either type of lipid vesicles. Similar results have been described by D'Antuono et al. (2000) in membranes and vesicles obtained from rat renal papillae.

\section{Construction and biochemical characterization of a phosphatidylglycerol mutant strain}

As shown in Fig. 1(a, b), the PG content in membranes of strain CSL2 grown in LBN increased, suggesting that this phospholipid might replace CL during the osmotic adaptation. To verify this hypothesis, the PGSA1 mutant, in which the pgsA gene is under the control of an inducible
Pspac promoter, was studied. This strain requires the presence of the inducer (IPTG) for its long-term viability (data not shown), indicating the importance of anionic phospholipids in the membranes of B. subtilis. This behaviour has also been reported by Matsumoto et al. (1999). Also, after $8 \mathrm{~h}$ incubation in the absence of IPTG, the cultures started to grow, indicating that the $p g s A$ mutation could be bypassed even in the presence of the LacI repressor. This result led us to choose early stationary phase cultures for the determination of the polar lipid composition and osmoresistance of the mutant strain induced with different IPTG concentrations. As shown in Table 3, the gradually increased content of the anionic phospholipids (PG and CL) was dependent on the IPTG concentration. Regarding the other two major zwitterionic phospholipids present in this strain, PE showed a slight increase at low IPTG concentrations $(0 \cdot 05-0 \cdot 1 \mathrm{mM})$ and then its value remained constant, whereas LysPG decreased with increasing IPTG concentrations (Table 3). The phospholipid composition of early stationary phase cultures from the wild-type strain was included as control in the experiments reported in Table 3. The presence of $\mathrm{PG}$ and $\mathrm{CL}$ in membranes of the PGSA1 mutant grown without IPTG could be due to an inducer-independent transcription of the pgsA gene and not to an increased lifetime of these phospholipids. DGDG accumulated in the membranes of strain PGSA1 grown in the absence of IPTG, but after addition of the inducer the content of this glycolipid decreased (data not shown), indicating a shift in the metabolism of lipid biosynthesis towards the synthesis of anionic phospholipids. The accumulation of DGDG observed in this mutant strain might be due to a change in the regulation of intracellular pools of cytidine $5^{\prime}$ diphosphate diacylglycerol (CDP-DAG), causing an increase in the synthesis of diacylglycerol, which is used as

Table 3. Phospholipid composition of B. subtilis strain PGSA1

Overnight cultures of strain PGSAl were grown in LB containing 0.375 M IPTG and diluted in LB containing the different IPTG concentrations as indicated; the wild-type strain (WT, strain YB886) was grown in LB. At the early stationary phase the cultures were collected and phospholipids quantified as indicated in Methods. Phospholipid contents are reported as percentage of total phospholipids (mol phosphorus). The values represent the mean $\pm \mathrm{SD}$ from three independent experiments.

\begin{tabular}{|c|c|c|c|c|}
\hline \multirow[t]{2}{*}{ IPTG (mM) } & \multicolumn{4}{|c|}{ Phospholipid } \\
\hline & LysPG & PE & PG & CL \\
\hline 0 & $31 \pm 6 \cdot 0$ & $53 \pm 3 \cdot 8$ & $11 \pm 3 \cdot 0$ & $3 \pm 0.9$ \\
\hline $0 \cdot 05$ & $25 \pm 4 \cdot 6$ & $60 \pm 4 \cdot 5$ & $12 \pm 0 \cdot 7$ & $3 \pm 1 \cdot 8$ \\
\hline $0 \cdot 1$ & $20 \pm 1 \cdot 6$ & $54 \pm 1 \cdot 1$ & $21 \pm 3 \cdot 6$ & $5 \pm 2 \cdot 0$ \\
\hline $0 \cdot 375$ & $8 \pm 1 \cdot 4$ & $52 \pm 2 \cdot 7$ & $33 \pm 3 \cdot 9$ & $7 \pm 0 \cdot 8$ \\
\hline WT & $15 \pm 2 \cdot 2$ & $36 \pm 1 \cdot 9$ & $34 \pm 2 \cdot 7$ & $15 \pm 3 \cdot 8$ \\
\hline
\end{tabular}


a substrate for DGDG biosynthesis. However, this assumption should be confirmed. The fatty acid analysis of this strain in the presence of different IPTG concentrations did not reveal any differences when compared with the wildtype (data not shown).

The osmoresistance assays performed with early stationary phase cultures clearly showed a direct correlation between the ability of PGSA1 strain to grow in the presence of $1.5 \mathrm{M}$ $\mathrm{NaCl}$ and the induction of the $p g s \mathrm{~A}$ gene: at the maximum pgsA expression ( $0.375 \mathrm{mM}$ IPTG) the osmoresistance of the PGSA1 mutant was $59.6 \%$ and the CL + PG content was $40 \%$. In similar assays, the wild-type strain showed $80 \%$ osmoresistance, while the anionic phospholipid content was $49 \%$ (Table 4).

\section{Transcriptional analysis of the c/sA (ywnE) and pgsA genes}

As stated above, the presence of anionic phospholipids is important for the osmotic stress response; therefore we analysed the transcriptional characteristics of the $c l s A$ and $p g s A$ genes by using transcriptional lac $Z$ fusions. The transcriptional analysis of the $c l s A$ gene was performed in both the mutant and wild-type context (strains CSL2 and CLS21) but no significant differences were observed, indicating that CL did not affect the regulation of the $c l s A$ gene, which is involved in CL synthesis (data not shown). The transcription of the $c l s A$ gene increased as cells reached the stationary phase, this induction being more significant (twofold increase) when the cultures were grown in the presence of $0.75 \mathrm{M} \mathrm{NaCl}$. These results agree with the biochemical data in which we observed an accumulation of $\mathrm{CL}$ in membranes of the wild-type strain not only upon entry into stationary phase but also during growth in LBN medium (Fig. 1a, b).

Table 4. Correlation between anionic phospholipid content in membrane and osmoresistance of PGSA1 and wild-type cultures

Strain PGSAl was grown in LB medium in the presence of the indicated IPTG concentrations; the wild-type (WT, strain YB886) was grown in LB. Samples were taken at the early stationary phase and used to determine both phospholipid content [percentage of $\mathrm{CL}+\mathrm{PG}$ (mol phosphorus)] and osmoresistance. The values represent the mean $\pm \mathrm{SD}$ of three independent experiments.

\begin{tabular}{|lcc|}
\hline IPTG $(\mathbf{m M})$ & PG $+\mathbf{C L}$ & Osmoresistance $(\%)^{*}$ \\
\hline 0 & $14 \pm 3 \cdot 9$ & $0 \cdot 16$ \\
$0 \cdot 05$ & $15 \pm 2 \cdot 5$ & $1 \cdot 5$ \\
$0 \cdot 1$ & $26 \pm 5 \cdot 6$ & $\mathrm{ND}$ \\
$0 \cdot 375$ & $40 \pm 4 \cdot 7$ & $59 \cdot 6$ \\
WT & $49 \pm 6 \cdot 5$ & $80 \cdot 0$ \\
\hline
\end{tabular}

${ }^{*}$ Osmoresistance was measured as indicated in Table 2 but all PGSA1 cultures were plated in medium containing 0.375 M IPTG. ND, Not determined.
The transcriptional analysis of the $p g s A$ promoter also indicated a slight induction of this gene at the early stationary phase (data not shown). Moreover, upon addition of increasing $\mathrm{NaCl}$ concentrations a twofold induction comparable to the $c l s A$ promoter activity was also observed.

\section{DISCUSSION}

In the YB886 genetic background, neither the $y$ wiE nor the ywjE mutant strains (CSL1 and CSL3) showed a deficiency in CL synthesis. In contrast, the interruption of the $y w n E$ gene (strain CSL2) resulted in a complete deficiency in CL synthesis. This mutant strain was completely unable to synthesize CL in the different media and growth phases assayed, thus supporting the proposal that $y w n E$ is a true $c l s A$ gene in B. subtilis YB886. It is important to mention that the phospholipid and glycolipid composition of B. subtilis strains YB886 (our wild-type context) and Marburg 168 (Kawai et al., 2004) are different; the PG content in YB886 was higher than that in Marburg 168. Furthermore, while in YB886 the $c l s A$ mutation did not produce a significant change in sporulation efficiency compared to the wild-type, the same mutation in the Marburg strain affected the sporulation process ( $50 \%$ reduction). Since we cannot rule out the possibility that the phospholipid assay that Kawai et al. (2004) used in their experiments could have been more sensitive than our assay, the overall differences discussed above are more likely to be due to differences in the genetic backgrounds of the strains used.

Regarding the ability to respond to an osmotic stress, the CSL2 mutant did not show an absolute defective phenotype. However, the extended lag observed in the presence of $1.5 \mathrm{M} \mathrm{NaCl}$, and the reduced growth, highlight the importance of the membrane CL content in the adaptation of $B$. subtilis to high salt concentrations. Since we observed an increased PG content in the CSL2 strain it is possible that in certain specific reactions that are dependent on the polar head group of the phospholipids, PG might substitute for the lack of CL, as has been described for Saccharomyces cerevisiae (Jiang et al., 2000) and E. coli (Matsumoto, 2001). Moreover, since changes in the lipid headgroup region of lipid bilayers could lead to modifications in the structure of transmembrane $\alpha$-helices of membrane proteins and as a result, changes in the packing of these helices, it is important to maintain the presence of charged lipids in the bilayer. This conclusion is also supported by the behaviour of the PGSA1 mutant and by the direct correlation between the anionic phospholipid (CL + PG) content and the osmoresponse observed in our work (Table 4). In addition, the failure of the PGSAl strain to survive in the absence of anionic lipids corroborates its importance for the integrity of the B. subtilis membrane. In fact, most of the genes related to envelope structure in B. subtilis, have been shown to be indispensable for viability (Kobayashi et al., 2003). The transcriptional activity of both $p g s A$ and $c s l A$ indicated that these genes were similarly regulated: the intensity of their 
promoter was similar and both showed a slight increase (twofold) at stationary phase or under osmotic upshift.

In this work we also demonstrated that the absence of CL in the $B$. subtilis membrane leads to additional modifications of other membrane components such as fatty acids and glycolipids, confirming that there is a tight cross-regulation between the different membrane components. Although a significant difference concerning the fatty acid composition of the CSL2 strain was observed when compared with the wild-type, we also showed that the overall changes produced in these membrane components as a consequence of an elevated $\mathrm{NaCl}$ concentration are similar in both strains, suggesting that these modifications might be a general mechanism of membrane adaptation to a high ionic strength.

Several lines of evidence suggest that the biophysical state of the membranes plays a key role in the regulation of some transporters, such as the mechanosensitive channels in $S$. cerevisiae (Gustin et al., 1988) and in E. coli (Moe \& Blount, $2005)$. Since the fluidity and functional properties of biological membranes are achieved by the full collaboration of proteins and lipids, we characterized the phase behaviour and interface properties of lipid vesicles and isolated membranes by studying the Laurdan's dipolar relaxation properties. In both the wild-type and the CSL2 strains, the lipid vesicles and membranes were in a liquid-ordered state and no phase coexistence or phase transitions were observed. However, our results clearly indicate that the lateral lipid packing of the vesicles and membranes varied due to the absence of CL. Another interesting feature is that the lateral lipid packing of the membranes of both strains increased when they were grown in LBN. This change could be related to the modifications described in this paper regarding the composition of their membranes (polar lipids and fatty acids). Since it is likely that different proteins require different amounts of water, an osmotic stress would potentially affect the activity of these proteins through changes in the hydration rate of the lipid surface and the membrane proteins as well (Poolman et al., 2002). Therefore, it is possible that the changes observed in the lipid hydration of the isolated membranes of the CSL2 mutant strain would affect the functional properties of specific membrane proteins, such as those involved in the osmotic response. The results in this paper allow us, for the first time, to emphasize the importance of anionic phospholipids in the B. subtilis membranes in withstanding high ionic strength.

One possible explanation for the major variations in lipids occurring during stress or growth phase is that they would influence the membrane structure, thus affecting the activity of enzymes involved in different processes such as sporulation (Dartois et al., 1997), osmosensing (Poolman et al., 2004) and modifications of protein function (Jensen \& Mouritsen, 2004). van der Heide et al. (2001), while studying the ionic regulation of the OpuA transporter of Lactococcus lactis in proteoliposomes containing different phospholipid composition, showed that only the charge present on the lipid headgroups influences the osmotic activation profile of this transporter. Likewise, Poolman et al. (2004) demonstrated that the activation threshold of the OpuA transporter, which is an indicator of osmosensor activity, depends on the fraction of anionic lipids present in the samples. These authors argued that this feature would indicate a general requirement for negatively charged lipids in several bacterial transporters even if they are not osmoregulated. In addition, in E. coli it has been demonstrated that the autophosphorylation of the sensor kinase $\mathrm{KdpD}$, which regulates the expression of the $k d p F A B C$ operon, is also dependent on negatively charged phospholipids (Stalkamp et al., 1999).

The presence of negatively charged lipids in the lipid bilayer of the B. subtilis cells grown in the presence of elevated $\mathrm{NaCl}$ concentrations would increase the negative charge of the surface of the membrane and therefore increase the concentration of positively charged ions or molecules close to the surface (Lee, 2004). This change in phospholipid content would be important for the osmotic adaptation of the cells, since the first response observed in both Gram-negative and Gram-positive bacteria is the uptake of $\mathrm{K}^{+}$into the cytoplasm (McLaggan et al., 1994; Whatmore \& Reed, 1990). Moreover, as was previously discussed, several transporters and regulatory proteins involved in the osmotic response in different bacteria are not only dependent on the fraction of anionic lipid but also on the physical state of the membranes. However, we cannot discount the possibility that this incorporation of negatively charged lipids would also change the affinities of membrane proteins for charged substrates as was suggested by Lee (2004).

\section{ACKNOWLEDGEMENTS}

We are very grateful to Drs F. Hullo, K. Matsumoto and P. Stragier for providing us with strains and plasmids. C.S. L. was a doctoral fellow of the Consejo Nacional de Investigaciones Científicas (CONICET) and part of this work was included in her PhD thesis. H. H., E. A. R. and C.S.R. are career investigators of the CONICET. This work was supported by grants of the University of Buenos Aires and CONICET, Argentina.

\section{REFERENCES}

Bagatolli, L. A. \& Gratton, E. (2001). Direct observation of lipid domains in free-standing bilayers using two-photon excitation fluorescence microscopy. J Fluoresc 11, 141-160.

Bartlett, G. P. (1959). Phosphorus assay in column chromatography. J Biol Chem 234, 466-468.

Bron, S. (1990). Plasmids. In Molecular Biological Methods for Bacillus, pp. 75-136. Edited by C. R. Harwood \& S. M. Cutting. Chichester, UK: Wiley.

Cao, M., Wang, T., Ye, R. \& Helman, J. D. (2002). Antibiotics that inhibit cell wall biosynthesis induce expression of the Bacillus subtilis $\sigma^{\mathrm{W}}$ and $\sigma^{\mathrm{M}}$ regulons. Mol Microbiol 45, 1267-1276. 
Catucci, L., Depalo, N., Lattanzio, V. M. T., Agostiano, A. \& Corcelli, A. (2004). Neosynthesis of cardiolipin in Rhodobacter sphaeroides under osmotic stress. Biochemistry 43, 15066-15072.

Correa, O. S., Rivas, E. A. \& Barneix, A. J. (1999). Cellular envelopes and tolerance to acid $\mathrm{pH}$ in Mesorhizobium loti. Curr Microbiol 38, 329-334.

Csonka, L. \& Hanson, A. (1991). Prokaryotic osmoregulation: genetics and physiology. Annu Rev Microbiol 45, 569-606.

D'Antuono, C., Fernandez-Tome, M. C., Sterin-Speziale, N. \& Bernik, D. L. (2000). Lipid-protein interactions in rat renal subcellular membranes: a biophysical and biochemical study. Arch Biochem Biophys 382, 39-47.

Dartois, V., Djavakhishvili, T. \& Hoch, J. A. (1997). KapB is a lipoprotein required for KinB signal transduction and activation for the phosphorelay to sporulation in Bacillus subtilis. Mol Microbiol 26, 1097-1108.

Glassker, E., Heuberger, E. H., Konings, W. N. \& Poolman, B. (1998). Mechanism of osmotic activation of the quaternary compound transporter (QacT) of Lactobacillus plantarum. J Bacteriol 180, 5540-5546.

Gustin, M. C., Zhou, X. L., Martinac, B. \& Kung, C. (1988). A mechanosensitive ion channel in the yeast plasma membrane. Science 242, 762-765.

Hanson, R. S. \& Phillips, J. A. (1981). Manual of Methods for General Bacteriology. Washington, DC: American Society for Microbiology.

Hirsch-Lerner, D. \& Barenholz, Y. (1999). Hydration of lipoplexes commonly used in gene delivery: follow up by Laurdan fluorescence changes and quantification by differential scanning calorimetry. Biochim Biophys Acta 1461, 47-57.

Horsburgh, M. J. \& Moir, A. (1999). Sigma M, an ECF RNA polymerase sigma factor of Bacillus subtilis 168, is essential for growth and survival in high concentrations of salt. Mol Microbiol 32, $41-50$.

Jensen, M. O. \& Mouritsen, O. G. (2004). Lipids do influence protein function - the hydrophobic matching hypothesis revisited. Biochim Biophys Acta 1666, 205-226.

Jiang, F., Ryan, M. T., Schlame, M., Zhao, M., Gu, Z., Klingenberg, M., Pfanner, N. \& Greenber, M. L. (2000). Absence of cardiolipin in the $c d r 1$ null mutant results in decreased mitochondrial membrane potential and reduced mitochondrial function. J Biol Chem 275, 22387-22394.

Kaneda, T. (1977). Fatty acids in the genus Bacillus: an example of branched-chain preference. Bacteriol Rev 41, 391-418.

Kappes, R. M., Kempf, B. \& Bremer, E. (1996). Three transport systems for the osmoprotectant glycine betaine operate in Bacillus subtilis: characterization of OpuD. J Bacteriol 178, 5071-5079.

Kates, M. (1986). Techniques of Lipidology: Isolation, Analysis and Identification of Lipids. New York: Elsevier.

Kawai, K., Shoda, M., Harashima, R., Sadaie, Y., Hara, H. \& Matsumoto, K. (2004). Cardiolipin domains in Bacillus subtilis Marburg membranes. J Bacteriol 186, 1475-1483.

Kempf, B. \& Bremer, E. (1998). Uptake and synthesis of compatible solutes as microbial stress responses to high-osmolality environments. Arch Microbiol 170, 319-330.

Kobayashi, K., Ehrlich, S. D., Albertini, A. \& 96 other authors (2003). Essential Bacillus subtilis genes. Proc Natl Acad Sci U S A 100, 4678-4683.

Kunst, F. \& Rapoport, G. (1995). Salt stress in an environmental signal affecting degradative enzyme synthesis in Bacillus subtilis. J Bacteriol 177, 2403-2407.

Lee, A. G. (2004). How lipids affects the activities of integral membrane proteins. Biochim Biophys Acta 1666, 62-87.
López, C. S., Heras, H., Ruzal, S. M., Sánchez-Rivas, C. \& Rivas, E. A. (1998). Variations on the envelope composition of Bacillus subtilis during growth in hyperosmotic medium. Curr Microbiol 36, 55-61.

López, C. S., Heras, H., Garda, H., Ruzal, S. M., Sánchez-Rivas, C. \& Rivas, E. A. (2001). Biochemical and biophysical studies of Bacillus subtilis. Int J Food Microbiol 55, 137-142.

López, C. S., Garda, H. \& Rivas, E. A. (2002). The effect of osmotic stress on the biophysical behavior of the Bacillus subtilis membrane studied by dynamic and steady-state fluorescence anisotropy. Arch Biochem Biophys 408, 220-228.

Lysenko, E., Ogura, T. \& Cutting, S. M. (1997). Characterization of the $f t s H$ gene of Bacillus subtilis. Microbiology 143, 971-978.

Machado, M. C., López, C. S., Heras, H. \& Rivas, E. A. (2004). Osmotic response in Lactobacillus casei ATCC 393: biochemical and biophysical characteristics of membrane. Arch Biochem Biophys 422, 61-70.

Martin-Verstraete, I., Debarbouillé, M., Klier, A. \& Rapoport, G. (1992). Mutagenesis of the Bacillus subtilis ' $-12,-24$ ' promoter of the levanase operon and evidence for the existence of an upstream activating sequence. J Mol Biol 226, 85-99.

Matsumoto, K. (2001). Dispensable nature of phosphatidylglycerol in Escherichia coli: dual roles of anionic phospholipids. Mol Microbiol 39, 1427-1433.

Matsumoto, K., Takahashi, H., Harashima, R. \& Hara, H. (1999). Membrane lipid synthesis in Bacillus subtilis Marburg. In 10th International Conference on Bacilli. June 27-July 1 1999, Grand Hotel Dino, Baveno, Italy, Abstract p. 72.

McLaggan, D., Naprstek, J., Buurman, E. T. \& Epstein, W. (1994). Interdependence of $\mathrm{K}^{+}$and glutamate accumulation during osmotic adaptation of Escherichia coli. J Biol Chem 269, 1911-1917.

Miller, J. H. (1992). A Short Course in Bacterial Genetics, pp. 72-74. Cold Spring Harbor, NY: Cold Spring Harbor Laboratory.

Moe, P. \& Blount, P. (2005). Assessment of potential stimuli for mechano-dependent gating of MscL: effects of pressure, tension, and lipid headgroups. Biochemistry 44, 12239-12244.

Parasassi, T. \& Gratton, E. (1995). Membrane lipid domains and dynamics as detected by Laurdan fluorescence. J Fluoresc 5, 59-69.

Parasassi, T., Di Stefano, M., Loeiro, M., Ravagnan, G. \& Gratton, E. (1994). Influence of cholesterol on phospholipid bilayers phase domains as detected by Laurdan fluorescence. Biophys J 66, 120-132.

Petersohn, A., Brigulla, M., Haas, S., Hoheisel, J. D., Volker, U. \& Hecker, M. (2001). Global analysis of the general stress response of Bacillus subtilis. J Bacteriol 83, 5617-5631.

Piuri, M., Sánchez-Rivas, C. \& Ruzal, S. M. (2003). Adaptation to high salt in Lactobacillus: role of peptides and proteolytic enzymes. J Appl Microbiol 95, 372-379.

Piuri, M., Sánchez-Rivas, C. \& Ruzal, S. M. (2005). Cell wall modifications during osmotic stress in Lactobacillus casei. J Appl Microbiol 98, 84-95.

Poolman, B., Blount, P., Folgering, J. H. A., Friesen, R. H. E., Moe, P. C. \& van der Heide, T. (2002). How do membrane proteins sense stress? Mol Microbiol 44, 889-902.

Poolman, B., Spitzer, J. J. \& Wood, J. M. (2004). Bacterial osmosensing: roles of membrane structure and electrostatics in lipid-protein and protein-protein interactions. Biochim Biophys Acta 1666, 88-104.

Price, C. W., Fawcett, P., Cérémonie, H., Su, N., Murphy, C. K. \& Youngman, P. (2001). Genome-wide analysis of the general stress response in Bacillus subtilis. Mol Microbiol 41, 757-774. 
Record, M. T., Jr, Courtenay, E. S., Cayley, D. S. \& Guttman, H. J. (1998). Responses of Escherichia coli to osmotic stress: large changes in amounts of cytoplasmic solutes and water. Trends Biochem Sci 23, 143-148.

Rivas, E. A. \& Luzzati, V. (1969). Polymorphisme des lipides polaires et des galactolipides de chloroplastes de maïs, en présence d'eau. J Mol Biol 41, 261-275.

Ruzal, S. \& Sánchez-Rivas, C. (1994). Physiological and genetic characterization of the osmotic stress response in Bacillus subtilis. Can J Microbiol 40, 140-144.

Ruzal, S. \& Sánchez-Rivas, C. (1998). Role of DegU in the osmotic stress response of Bacillus subtilis. Curr Microbiol 37, 368-372.

Ruzal, S., López, C., Rivas, E. \& Sánchez-Rivas, C. (1998). Osmotic strength blocks sporulation at stage II by impeding activation of early sigma factors in Bacillus subtilis. Curr Microbiol 36, 75-79.

Sánchez-Rivas, C. \& Bohin, J. P. (1983). Cardiolipin content and protoplast fusion efficiency in Bacillus subtilis. FEMS Microbiol Lett 19, 137-141.

Schaeffer, P., Millet, J. \& Aubert, J. P. (1965). Catabolic repression of bacterial sporulation. Proc Natl Acad Sci U S A 54, 704-711.

Stalkamp, I., Dowhan, W., Altendorf, K. \& Jung, K. (1999). Negatively charged phospholipids influence the activity of the sensor kinase KdpD of Escherichia coli. Arch Microbiol 172, 295-302.

Steil, L., Hoffmann, T., Budde, I., Volker, U. \& Bremer, E. (2003). Genome-wide transcriptional profiling analysis of adaptation of Bacillus subtilis to high salinity. J Bacteriol 185, 6358-6370.
Thackray, P. D. \& Moir, A. (2003). SigM, an extracytoplasmic function sigma factor of Bacillus subtilis, is activated in response to cell wall antibiotics, ethanol, heat, and superoxide stress. J Bacteriol 185, 3491-3498.

Vagner, V., Dervyn, E. \& Erlich, D. (1998). A vector for systematic gene inactivation in Bacillus subtilis. Microbiol 144, 3097-3104.

van der Heide, T., Stuart, M. C. A. \& Poolman, B. (2001). On the osmotic signal and osmosensing mechanism of an $\mathrm{ABC}$ transport system for glycine betaine. EMBO J 20, 7022-7032.

von Blohn, C., Kempf, B., Capees, R. M. \& Bremer, E. (1997). Osmostress response in Bacillus subtilis: characterization of a proline uptake system (OpuE) regulated by high osmolarity and the alternative transcription factor sigma B. Mol Microbiol 25, 175-187.

Whatmore, A. M. \& Reed, R. H. (1990). Determination of turgor pressure in Bacillus subtilis: a possible role of $\mathrm{K}^{+}$in turgor regulation. J Gen Microbiol 136, 2521-2526.

Whatmore, A. M., Chudek, J. A. \& Reed, R. H. (1990). The effects of osmotic upshock on the intracellular solute pools of Bacillus subtilis. J Gen Microbiol 136, 2527-2535.

Wood, J. M. (1999). Osmosensing by bacteria: signals and membranebased sensors. Microbiol Mol Biol Rev 63, 230-262.

Yan, D., Ikeda, T. P., Shauger, A. E. \& Kustu, S. (1996). Glutamate is required to maintain the steady state potassium pool in Salmonella typhimurium. Proc Natl Acad Sci U S A 93, 6527-6531.

Yasbin, R., Fields, P. \& Anderson, P. (1980). Properties of Bacillus subtilis 168 derivatives freed of their natural prophages. Gene 12, 155-169. 